\title{
The First Febrile Seizure: An Updated Study for Clinical Risk Factors
}

\author{
Farhad Heydarian ${ }^{1,{ }^{*}}$, Elham Bakhtiari ${ }^{2}$, Sara Yousefi ${ }^{3}$ and Mohammad Heidarian ${ }^{4}$ \\ ${ }^{1}$ Department of Pediatrics, Faculty of Medicine, Mashhad University of Medical Sciences, Mashhad, Iran \\ ${ }^{2}$ Research Center for Patients Safety, Mashhad University of Medical Sciences, Mashhad, Iran \\ ${ }^{3}$ Mashhad University of Medical Sciences, Mashhad, Iran \\ ${ }^{4}$ California State University, Hayward, California, United States \\ "Corresponding author: Professor of Pediatrics, Department of Pediatrics, Mashhad University of Medical Sciences, Mashhad, Iran. Tel: +98-5138012469, Fax: +98-5138417451, \\ Email: heydarianf@mums.ac.ir
}

Received 2018 April 23; Revised 2018 September 06; Accepted 2018 September 13.

\begin{abstract}
Objectives: The study aimed to determine major clinical risk factors for seizures in febrile children aged 6 - 60 months. Methods: One hundred seventy five febrile children aged 6 - 60 months with or without seizure were studied. Demographic, family history of epilepsy, family history of febrile seizure, type of parturition, smoking in pregnancy, infection diseases, and other predisposing factors were analyzed.

Results: Among the 175 children (97 patients with febrile seizure as the case group and 78 febrile patients without seizure as the control group), 90 cases (51.4\%) were female and 85 cases (48.6\%) were male with the average age of $23.02 \pm 17.78$ months. There was no significant difference between the groups in age and $\operatorname{sex}(\mathrm{P}=0.05)$. Seizure was simple in 73 patients $(75.3 \%)$ and complex in 24 patients (24.7\%). There was a family history of febrile seizure in 24 patients of the case group (24.7\%) and one patient of the control group $(1.3 \%)(\mathrm{P}<0.001)$. There was a family history of epilepsy in $17(17.5 \%)$ and three $(3.8 \%)$ patients in the case and control groups, respectively $(\mathrm{P}=0.005)$. The upper respiratory infection was detected more in the case group $(\mathrm{P}<0.001) .62$ patients $(63.9 \%)$ of the case group and 30 patients (40\%) of the control group were delivered via cesarean sections $(\mathrm{P}=0.002)$. The family history of epilepsy, cesarean section, family history of FS, and upper respiratory tract infection were recognized as the risk factors for convulsion in febrile children with 11.35, 3.43, 25.33, and 6.26 odds ratios, respectively.

Conclusions: The family history of epilepsy, cesarean section, family history of febrile seizure, and upper respiratory tract infection are the most common risk factors for seizure in febrile children.
\end{abstract}

Keywords: Seizures, Fever, Child, Risk Factor

\section{Background}

Seizures are the common cause of emergencies in children (1). Febrile seizure (FS) is the most common type of seizures affecting $2-5 \%$ of children aged $6-60$ months. This seizure is typically associated with a high fever during children's early life. The FS peaks in the second year of life $(1,2)$. Both genetics and environmental factors are involved (3). Some studies suggested that prenatal problems including asphyxia, premature birth, and parturition complications also are involved $(4,5)$. FS is often considered as benign. It is accepted that most children with FS have normal health and development after the FS incident (6). But, it has been recently reported that some children with febrile seizure may have recurrent FS or developed epilepsy (7-9).

\section{Objectives}

Considering the importance of FS in children and future unpleasant outcomes, the present study was con- ducted to evaluate the main risk factors for seizure in hospitalized febrile children aged 6 - 60 months.

\section{Methods}

The present descriptive-analytical study was performed in Ghaem Hospital, affiliated to Mashhad University of Medical Sciences, Mashhad, Iran, from 2008 to 2015. 97 children aged 6 - 60 months with first febrile seizure as the case group were compared with 78 febrile children without seizure as the control group. The groups were matched for age and sex. All patients in the case group showed the classic criteria of FS. Patients in the control group were febrile but without a seizure. The axillary body temperature was measured according to the standard protocol. The diagnosis of FS was documented based on the clinical symptoms by a pediatrician. Patients with meningitis, encephalitis, brain hemorrhage, neurodevelopmental delay, febrile status epilepticus, and history of trauma were excluded. 


\subsection{Analysis}

Statistical analysis was performed using SPSS version 19 program for windows (SPSS Institute, Inc., Chicago, IL, USA). All experimental values are presented as means \pm SD or frequency percentages. The Chi-squared test was used to screen the associations between symptoms and potential risk factors. Logistic Regression was performed to determine the risk factors. P values of less than 0.05 were considered statistically significant.

\section{Results}

Of the 175 children, $48.6 \%(n=85)$ were male and $51.4 \%$ $(n=90)$ were female. In the case group, $42.3 \%(n=41)$ were female and $57.7 \%(n=56)$ were male. In the control group, $56.4 \%(n=44)$ were female and $43.6 \%(n=34)$ were male. The difference between the groups in sex was not significant $(P=0.06)$. The average age of the patients was 23.02 \pm 17.78 months. The average age of patients in the case and control groups was $23.64 \pm 16.25$ and $22.25 \pm 19.59$ months, respectively $(P=0.6)$. In the case group, $57.7 \%(n: 56)$ were male and 42.3\% (n: 41) were female. There were 43.6\% (n: 34) male and $56.4 \%$ ( $\mathrm{n}: 44)$ female patints as controls ( $\mathrm{P}=$ $0.06)$.

The type of seizure in 73 patients (75.3\%) was simple and in 24 patients (24.7\%) was complex. $55 \%$ of the complex seizures occurred in 1-2-year-old patients.

The average weight of the patients in the case and control groups was $11.21 \pm 2.53$ and $10.02 \pm 3.95 \mathrm{~kg}$, respectively $(\mathrm{P}=0.051)$. The average birth weight in the case and control groups was $3137.93 \pm 553.57$ and $3164.74 \pm 452.41$ grams, respectively $(\mathrm{P}=0.73)$. The birth weight in 12 patients $(13 \%)$ in the case group and nine patients (11.5\%) in the control group was less than 2500 grams $(\mathrm{P}=0.76)$. There was a family history of FS in 24 patients (24.7\%) and one patient (1.3\%) of the case and control groups, respectively $(\mathrm{P}<0.001)$. There was a family history of epilepsy in 17 (17.5\%) and three (3.8\%) patients in the case and control groups, respectively $(\mathrm{P}=0.005)$. The patients were classified according to infections including upper respiratory infection (URTI), gastroenteritis, pneumonia, urinary tract infection (UTI), and other infections. There was a significant difference in the frequency of infections in the case and control groups $(\mathrm{P}<$ 0.001) (Figure 1).

The body temperature in 48 patients ( $49.5 \%$ ) of the case group and 32 patients (41\%) of the control group was more than $38.5^{\circ} \mathrm{C}(\mathrm{P}=0.26) .62$ patients $(63.9 \%)$ in the case group and 30 patients $(40 \%)$ in the control group were delivered via cesarean sections $(\mathrm{P}=0.002)$. There was a history of maternal antibiotics use in pregnancy in nine patients (9.4\%) of the case group and eight patients (10.3\%) of the control group $(\mathrm{P}=0.84)$. There was a history of maternal smoking in pregnancy in six patients (8.7\%) of the case group and one patient (3.8\%) of the control group $(\mathrm{P}=0.6)$.

The results of logistic regression showed that the family history of epilepsy as 11 times, cesarean section as 3.5 times, family history of FS as 25 times, and URTI as 6 times are involved as the main risk factors for seizure in febrile children. The results are shown in Table 1.

\begin{tabular}{lcc}
\hline Table 1. Risk Factors for Seizure in 175 & Febrile Children with or Without & Seizure \\
\hline Risk Factors & Odds Ratio & P Value \\
\hline Family history of epilepsy & 11.35 & 0.005 \\
Family history of FS & 25.33 & 0.003 \\
\hline Cesarean section & 3.43 & 0.005 \\
Upper respiratory tract infection & 6.26 & $<0.001$ \\
\hline
\end{tabular}

\section{Discussion}

175 febrile children aged 6 - 60 months were studied for the risk factors of seizures. Patients were classified as the case (with first seizure) and control (without seizure) groups. The average age was $23.02 \pm 17.78$ months. The family history of epilepsy, FS, URTI, and cesarean section were recognized as major risk factors. There was no significant association between antibiotic use in pregnancy, sex, maternal smoking, and high fever, and seizure occurrence.

The risk factors for seizure in febrile children were investigated in some studies $(4,10-12)$. In a study on 88 febrile children aged less than 5 years with or without fever URTI, the family history of FS, prematurity, gestation problems, family history of epilepsy, and parturition complications were recognized as risk factors (10). In another study on 160 febrile children with or without first seizure, it was concluded that gender, family history of febrile seizure, high fever, and short breastfeeding period were involved as seizure risk factors (11). Berg reported that gastroenteritis and smoking during pregnancy are the important risk factors for the first febrile seizure (4). In our study, smoking and drug use in pregnancy were not the risk factors for FS. It may be due to the difference in the type and duration of using these agents in pregnancy. In a study, the family history of convulsion was emphasized (13) and in another study, the developmental disorder was recognized as the risk factors of the first febrile seizure (14). In a study, the genetic role in the occurrence of febrile seizure was highlighted (3). There are a few studies regarding cesarean section and URTI as the risk factors of seizure $(15,16)$. In the present study, we showed that the cesarean section and URTI are involved as the risk factor of first febrile seizure with odds ratios of 3.43 and 1.83 , respectively. 


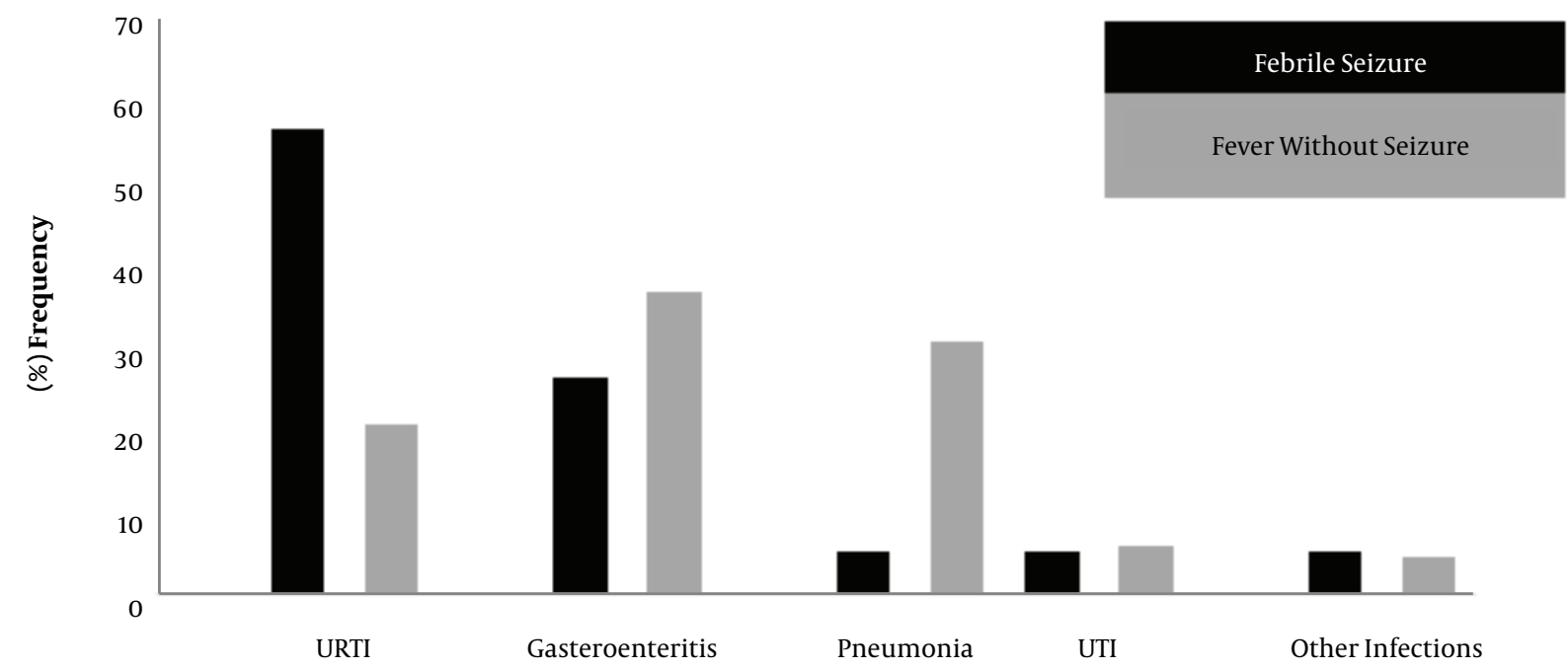

Figure 1. The frequency of infections in 175 febrile children with or without a seizure. Abbreviations: URTI, upper respiratory tract infection; UTI, urinary tract infection.

Regarding different studies evaluating the first febrile seizure, it is obvious that a range of various factors could be considered as the risk factors of first febrile seizure. Therefore, the identification of risk factors in each community should be considered as a major medical necessity. Although FS is accompanied by a good prognosis in most patients, the risk of epilepsy development in the presence of some risk factors including the family history of FS and epilepsy may rise up to $9 \%$ (11). Therefore, attempt to recognize the risk factors and prevent from them is effective for the prevention of seizure in febrile children.

\subsection{Conclusion}

According to our data, the family history of FS, epilepsy, cesarean section, and URTI are the main risk factors for seizure in febrile children. Accordingly, preventive measures may lead to a decreased febrile seizure.

\subsection{Limitations}

Breastfeeding period information was not available.

\section{Footnotes}

Authors' Contribution: Conception or design: Farhad Heydarian and Sara Yousefi. Acquisition and analysis: Elham Bakhtiari and Mohammad Heidarian. Drafting the work: Farhad Heydarian, Elham Bakhtiari, Sara Yousefi and Mohammad Heidarian. Final approval: Farhad Heydarian, Elham Bakhtiari, Sara Yousefi and Mohammad Heidarian.
Conflict of Interests: The authors declare that there is no conflict of interest.

Ethical Considerations: This study was approved by Ethical Committee of Mashhad University of Medical Sciences, Mashhad, Iran.

Financial Disclosure: None declared.

Funding/Support: This study was granted by Mashhad University of Medical Sciences, Mashhad, Iran.

\section{References}

1. Heydarian F, Ashrafzadeh F, Kam S. Simple febrile seizure: The role of serum sodium levels in prediction of seizure recurrence during the first 24 hours. Iran J Child Neurol. 2009;3(2):31-4. doi: 10.22037/ijcn.v3i2.1269.

2. Fetveit A. Assessment of febrile seizures in children. Eur J Pediatr. 2008;167(1):17-27. doi:10.1007/s00431-007-0577-x. [PubMed:17768636].

3. Nakayama J. Progress in searching for the febrile seizure susceptibility genes. Brain Dev. 2009;31(5):359-65. doi: 10.1016/j.braindev.2008.11.014. [PubMed: 19201561].

4. Berg AT, Shinnar S, Shapiro ED, Salomon ME, Crain EF, Hauser WA. Risk factors for a first febrile seizure: A matched case-control study. Epilepsia. 1995;36(4):334-41. [PubMed: 7541745].

5. Abuekteish F, Daoud AS, al-Sheyyab M, Nou'man M. Demographic characteristics and risk factors of first febrile seizures: A Jordanian experience. Trop Doct. 2000;30(1):25-7. doi: 10.1177/004947550003000113. [PubMed:10842518].

6. Seinfeld DS, Pellock JM. Recent research on febrile seizures: A review. J Neurol Neurophysiol. 2013;4(165). doi: 10.4172/2155-9562.1000165. [PubMed: 25383238]. [PubMed Central: PMC4220240].

7. Lee SH, Byeon JH, Kim GH, Eun BL, Eun SH. Epilepsy in children with a history of febrile seizures. Korean J Pediatr. 2016;59(2):74-9. doi: 10.3345/kjp.2016.59.2.74. [PubMed: 26958066]. [PubMed Central: PMC4781735]. 
8. Vestergaard M, Pedersen CB, Sidenius P, Olsen J, Christensen J. The long-term risk of epilepsy after febrile seizures in susceptible subgroups. Am J Epidemiol. 2007;165(8):911-8. doi: 10.1093/aje/kwk086. [PubMed: 17267419].

9. Maia C, Moreira AR, Lopes T, Martins C. Risk of recurrence after a first unprovoked seizure in children. J Pediatr (Rio J). 2017;93(3):281-6. doi: 10.1016/j.jped.2016.07.001. [PubMed: 27686587].

10. Abd Ellatif F, El Garawany H. Risk factors of febrile seizures among preschool children in Alexandria.J Egypt Public Health Assoc. 2002;77(12):159-72. [PubMed: 17219896].

11. Mahyar A, Ayazi P, Fallahi M, Javadi A. Risk factors of the first febrile seizures in Iranian children. Int J Pediatr. 2010;2010:862897. doi: 10.1155/2010/862897. [PubMed: 20652051]. [PubMed Central: PMC2905933].

12. Veisani Y, Delpisheh A, Sayehmiri K. Familial history and recurrence of febrile seizures; a systematic review and meta-analysis. Iran
J Pediatr. 2013;23(4):389-95. [PubMed: 24427491]. [PubMed Central: PMC3883367].

13. Veisani Y, Delpisheh A, Sayehmiri K. Predictors of recurrent febrile seizures in Iranian children. Zahedan J Res Med Sci. 2013;15(9):1-5.

14. Huang CC, Wang ST, Chang YC, Huang MC, Chi YC, Tsai JJ. Risk factors for a first febrile convulsion in children: A population study in southern Taiwan. Epilepsia.1999;40(6):719-25. [PubMed:10368069].

15. Gontko-Romanowska K, Zaba Z, Panienski P, Steinborn B, Szemien $M$, Lukasik-Glebocka $M$, et al. The assessment of risk factors for febrile seizures in children. Neurol Neurochir Pol.2017;51(6):454-8. doi 10.1016/j.pjnns.2017.07.011. [PubMed: 28803641].

16. Sidenvall R, Heijbel J, Blomquist HK, Nystrom L, Forsgren L. An incident case-control study of first unprovoked afebrile seizures in children: A population-based study of pre-and perinatal risk factors. Epilepsia. 2001;42(10):1261-5. [PubMed: 11737160]. 